\title{
FLAT-FIELD PLANAR STRUCTURES DESIGNED FOR SMALL AND LARGE ASPECT RATIOS*
}

\author{
D. Yu, A.V. Smirnov, Y. Luo, DULY Research Inc., Rancho Palos Verdes, CA 90275
}

\begin{abstract}
We consider novel designs that provide field flatness of fundamental mode in transverse dimensions of planar $\mathrm{rf}$ structures. These designs include waveguides with slotted irises and/or dielectric loaded side openings. The specially modified, advanced planar structures can be produced with available technology for a number of purposes. Highaspect ratio structures are suitable for mm-wave generation in sheet-beam klystrons (SBK) and for industrial, medical and research linacs. Small aspect-ratio planar structures having "flat" impedance in central area may be applicable to linear colliders. Impedance flatness better than 1/1,000 over half of aperture cross-section is demonstrated in simulations done for sample structures.
\end{abstract}

\section{INTRODUCTION}

Efficient, high-gradient acceleration schemes are essential for future linear colliders and advanced commercial electron accelerators. Many of the rf structure based schemes operate at high frequencies ( $\mathrm{Ka}$ to $\mathrm{W}$ bands) in order to achieve high gradients and high wallplug-to-acceleration efficiency. Examples of several linear collider designs scaled in frequency and energy from the NLC were worked out a few years ago by Perry Wilson et al [1]. To meet these $\mathrm{rf}$ power requirements and gradients, parameters of a sheet beam klystron (SBK) can be easily scaled to higher frequencies from an X-band structure designed by DULY Research [2,3].

Planar mm-wave structures are more suitable than traditional axially symmetric structures if microfabrication techniques are used [4]. Planar rf structures with high-aspect ratio flat beam can be used for both acceleration and $\mathrm{rf}$ generation purposes. To interact efficiently with a flat beam the planar rf structure should be designed with a constant effective longitudinal electric field in the larger transverse dimension (say, horizontal) of the accelerating gap. Such structures having 'flat' impedance profile in transverse direction are referred to here as flat-field structures. Feasibility of flat-field highaspect ratio cavities and waveguides as well as the main approaches for designing are demonstrated in ref. [5].

We introduced earlier [5] additional parameters for characterization flat-field periodic structures with different aspect ratios, aperture dimensions, operating frequencies $f=2 \pi c / \lambda$, and phase advance per cell $\theta$. Here we use generalized shunt impedance $R_{g}$ defined as $R_{g} / Q=S \cdot(R / Q) \cdot(a / \lambda)$, where $R$ and $Q$ are standard $Q$-factor and shunt impedance correspondingly, $2 a$ is the vertical gap and $S$ is the effective aspect ratio of the structure aperture.

The main problem in designing effective flat-field structures is modifying boundary conditions in order to provide field flatness along with high $R_{g} / Q$ value. In other words, the impedance profile across the aperture should be close to rectangular shape. In addition, manufacturing requirements represent an important limitation to the geometries under study. For common microfabrication processes, the structure should be etched with a single Xray mask resulting in uniform cavity depth and arbitrary cavity shape.

Computations and design optimizations were made with the code GdfidL [6] for ultrarelativistic beam.

\section{HIGH-ASPECT RATIO STRUCTURES}

We distinguish here high aspect ratio $(>8)$ and small aspect ratio $(<5)$ structures as requiring different approaches to implement the essential flatness. The main parameters for the periodic structure designs are given in Tables 1 and 2 correspondingly. Some of the structures contain dielectric material $\mathrm{SiC}$ with permeability $\varepsilon \approx 12.8$ (for X-band). Most of the designs have iris widths less than the cavity width. The continuous side space provides magnetic side coupling.

The main difference between M1 and M2 structures is the rod length: in the M1 variant it is shorter than half the gap, whereas in the M2 variant it is a continuous rod throughout the structure.

Asymmetrical configuration illustrated in Fig. 5 (type M5) looks like biperiodic structure. However, the asymmetry is introduced only to provide flatness by longitudinally interchanging the magnetic and electrical side coupling. Some asymmetry of field profile (Fig. 6) can be partially compensated in terms of shunt impedance of two adjacent $(\pi / 2)$ sub-cells.

One of the most practical structures with dielectric is shown in Fig. 6. It contains a long dielectric slab filling the side openings between plates. At a certain width of the slab a good field flatness can be achieved.

\footnotetext{
* This work is supported by DOE SBIR No. DE-FG03-96ER82213.
} 
Table 1: Flat-Field Planar Periodic Structures with high aspect ratio

\begin{tabular}{|l|l|l|l|l|l|l|l|l|}
\hline Type & \multicolumn{1}{|c|}{ Description } & $\begin{array}{c}f, \\
\mathrm{GHz}\end{array}$ & $\operatorname{mode} \theta$ & \multicolumn{1}{c|}{$\begin{array}{c}r / Q, \\
\mathrm{k} \Omega / \mathrm{m}\end{array}$} & \multicolumn{1}{|c|}{$\beta_{\mathrm{gr}}$} & $R_{g} / Q, \Omega$ & $S$ \\
\hline \hline M1 & short rod & 11.78 & $\pi / 2$ & 6050 & 2.965 & 0.06 & 21.6 & 11.5 \\
\hline M1 & short rod & 12.12 & $2 \pi / 3$ & 5480 & 2.834 & 0.05 & 29.3 & 11.5 \\
\hline M1 & short rod & 11.81 & $\pi$ & 6030 & 2.047 & 0 & 31.1 & 12 \\
\hline M2 & long rod \& T-end iris & 12.15 & $2 \pi / 3$ & 5753 & 2.76 & 0.074 & 25.9 & 11.4 \\
\hline M2 & long rod \& T-end iris & 11.43 & $2 \pi / 3$ & 5789 & 3.835 & 0.02 & 37.1 & 19 \\
\hline M3 & profiled iris thickness & 12.4 & $\pi / 2$ & 4529 & 3.08 & 0.068 & 16.7 & 8.94 \\
\hline M4 & long diel. strip in side space & 12.19 & $\pi / 2$ & 4896 & 2.89 & 0.1 & 22.4 & 12.6 \\
\hline M5 & snake-wise asymmetric & 12.08 & $\pi$ & 6074 & 1.732 & 0 & 21.7 & 8.84 \\
\hline M6 & interlayer diel. slab $(\varepsilon=12.8)$ & 11.44 & $2 \pi / 3$ & 5407 & 4.587 & 0.0088 & 29.1 & 11.9 \\
\hline
\end{tabular}

Table 2: Flat-Field Small-Aspect-Ratio Planar TW Structures $(\theta=2 \pi / 3)$

\begin{tabular}{|l|l|l|l|l|l|l|l|}
\hline Type & \multicolumn{1}{|c|}{ Description } & $\begin{array}{c}f, \\
\mathrm{GHz}\end{array}$ & \multicolumn{1}{|c|}{$\begin{array}{c}r / Q, \\
\mathrm{k} \Omega / \mathrm{m}\end{array}$} & $a / \lambda$ & $R_{g} / Q, \Omega$ & $S$ \\
\hline \hline S1 & side slot \& T-end iris & 11.86 & 3433 & 7.04 & 0.063 & 11.65 & 3.12 \\
\hline S2 & T-end, profiled iris \& side openings & 11.91 & 3524 & 8.274 & 0.063 & 16.39 & 3.75 \\
\hline M6 & interlayer dielectric strip $(\varepsilon=14.4)$ & 2.851 & 9300 & 1.732 & 0.066 & 13.28 & 3.6 \\
\hline S3 & No side space; side diel. plates in every cell & 22.4 & 3153 & 9.1 & 0.091 & 5.7 & 2.3 \\
\hline S4 & Side space only & 11.61 & 4731 & 10.645 & 0.061 & 6.96 & 1.25 \\
\hline S5 & Central and side slots + side openings & 11.91 & 3442 & 9.49 & 0.0625 & 9.34 & 1.88 \\
\hline
\end{tabular}

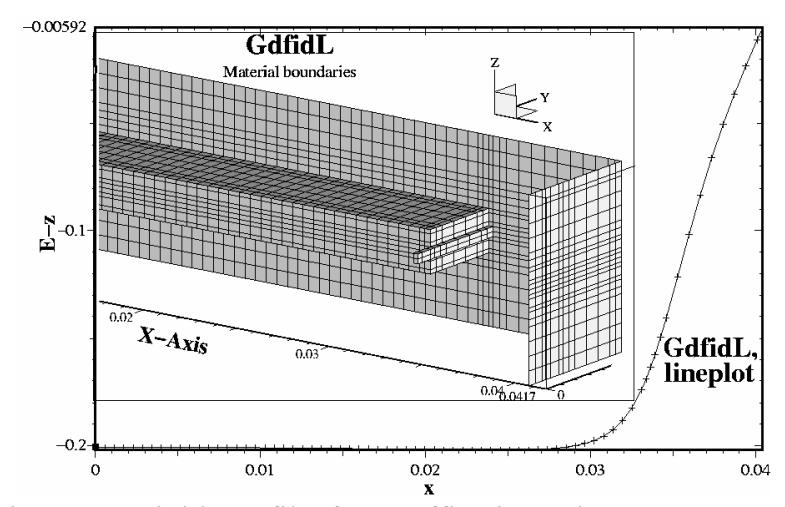

Figure 1: Field profile for muffin-tin $2 \pi / 3$ TW structure with side slot and short rod (M1).

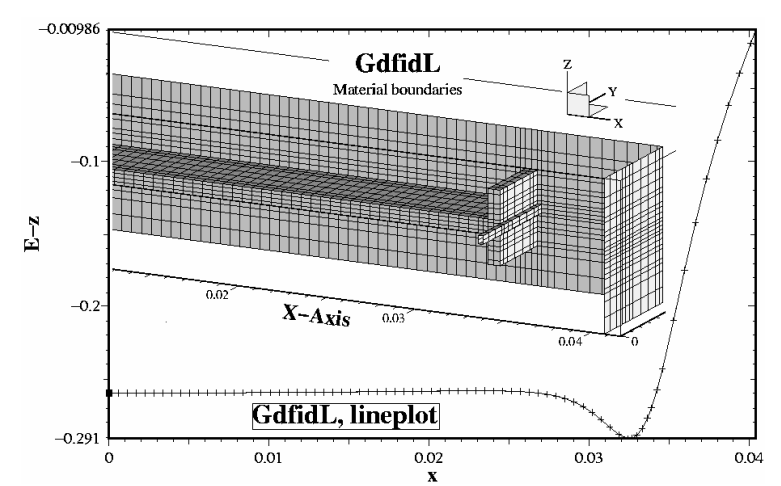

Figure 2: Field profile for muffin-tin $2 \pi / 3 \mathrm{TW}$ structure with T-end, shaped irises and rods extending across the gap (M2).

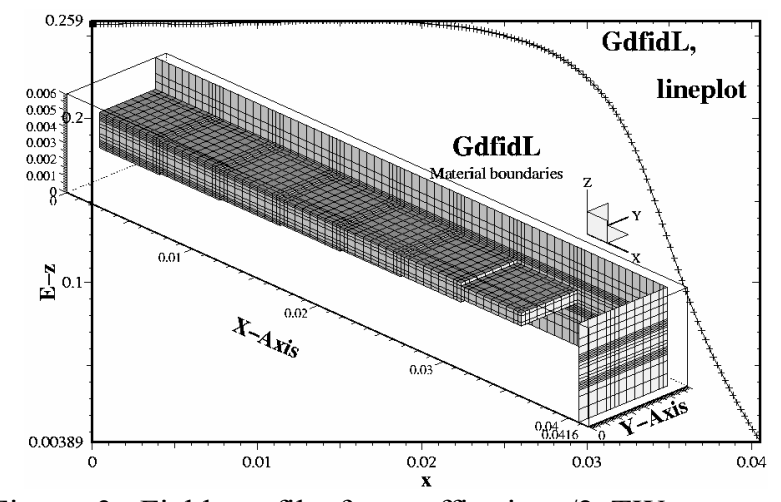

Figure 3: Field profile for muffin-tin $\pi / 2 \mathrm{TW}$ structure with profiled iris thickness (M3).

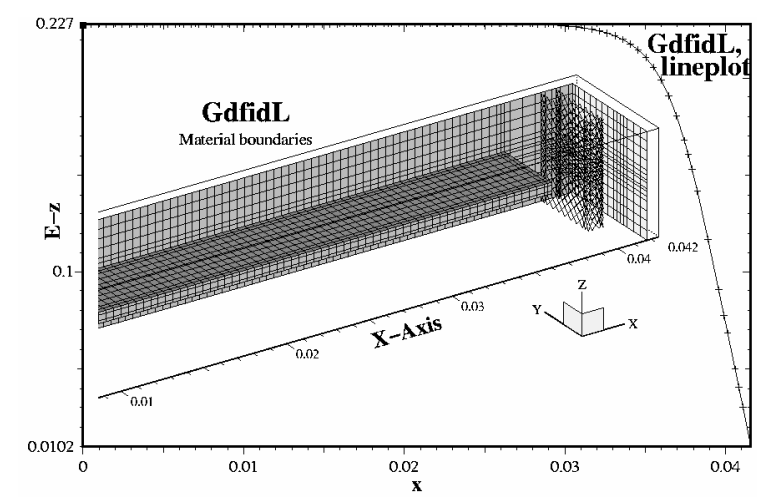

Figure 4: Field profile for muffin-tin $\pi / 2$ TW structure with dielectric slab (M4). 


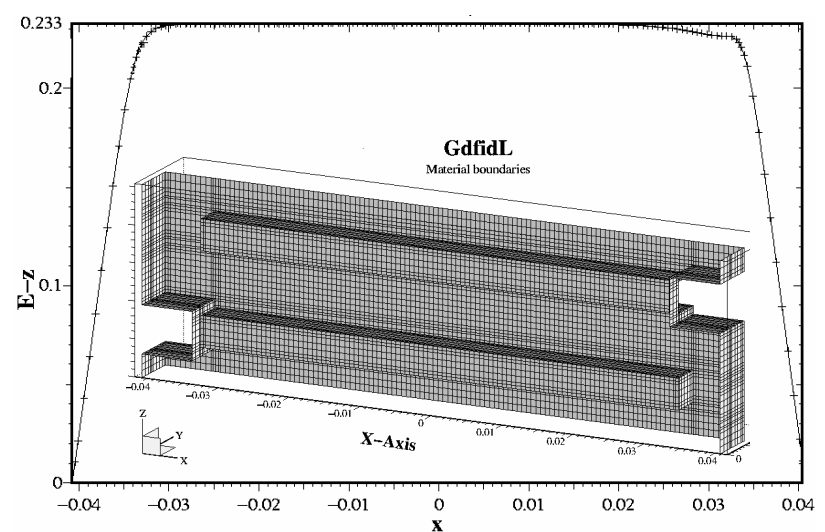

Figure 5: Field profile between irises for M5 type structure.

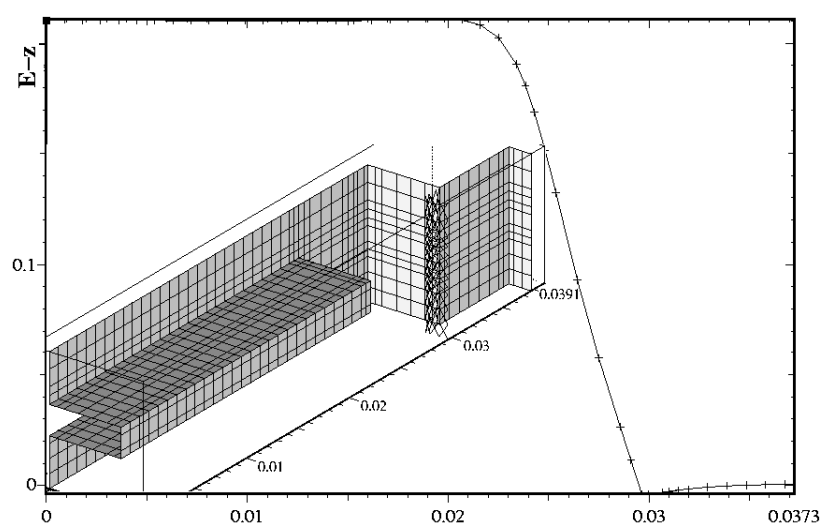

Figure 6: Field profile for muffin-tin $2 \pi / 3$ TW structure with side space and dielectric slab in side openings (M6).

The field plots depicted in Figs. 1-6 are given for $z=0$ coordinate (i.e. between irises). For $z \neq 0$ the plots are different. Nevertheless, the 2D profile of the impedance across the aperture can be made 'resonantly' flat with the design optimization. As an example the impedance flatness is characterized in Fig. 7 for the M6 design.

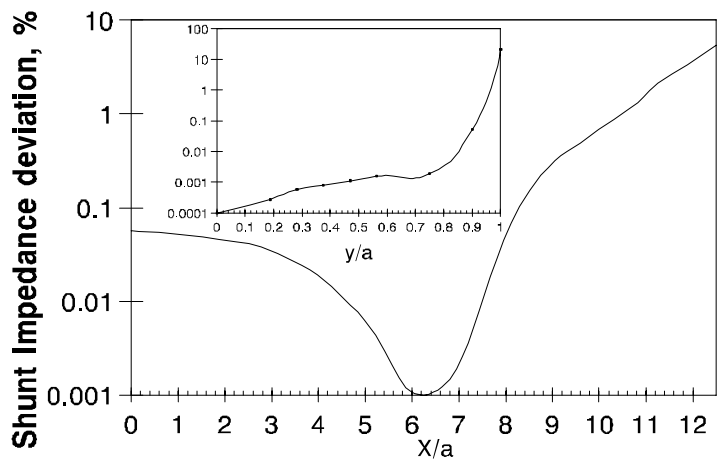

Figure 7: Relative deviation of the shunt impedance in $\mathrm{XOZ}$ plane and YOZ plane (inset) for the variant M6.

\section{SMALL-ASPECT RATIO STRUCTURES}

Small-aspect ratio structures listed in Table 2 can have significantly reduced 'quadrupole' effect of the fundamental (accelerating) mode. The S1, S2, S4 designs use combinations of previously considered approaches. The S3 configuration is based on classical muffin-tin with dielectric layer covering vertical side cavity walls inside each cell. The S5 configuration illustrated in Fig. 8 has a central slot in the iris. This slot plays a dual role: to provide field flatness and dipole mode suppression.

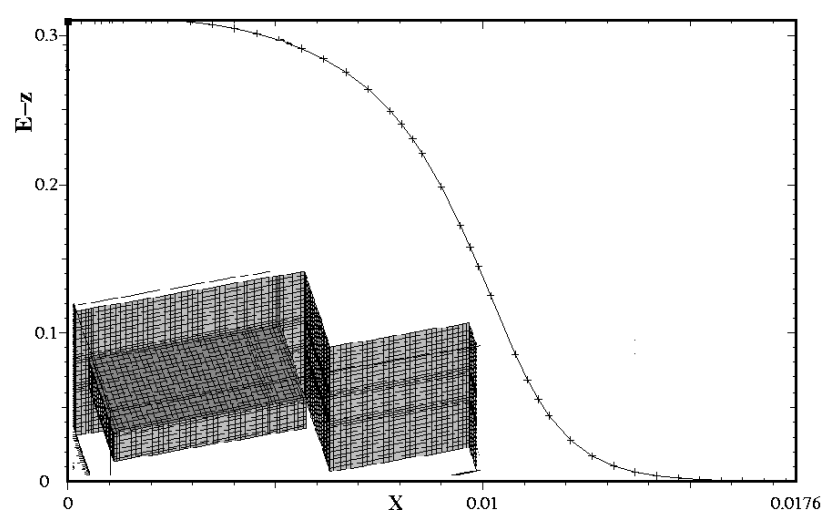

Figure 8: Field profile $(z=0)$ in $\mathrm{XOZ}$ plane for smallaspect ratio TW structure with central slot in the iris, side space and side openings (S5).

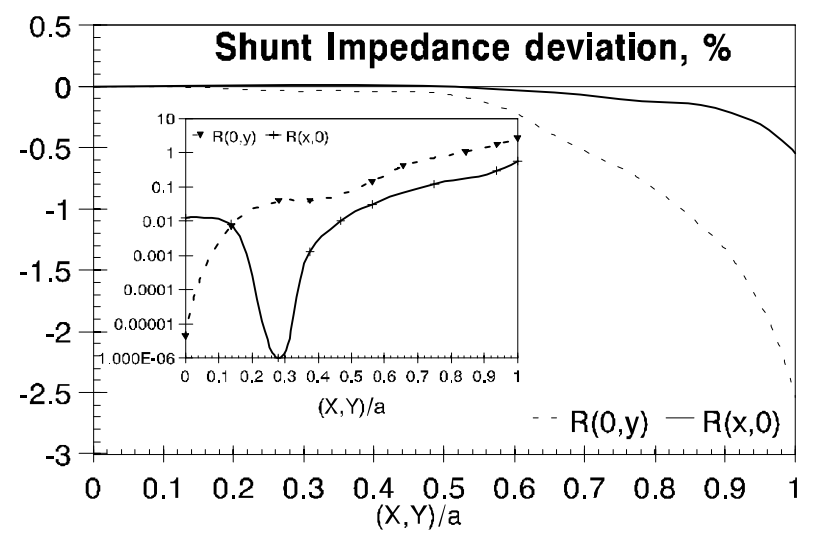

Figure 9: Relative deviation of the shunt impedance in $\mathrm{XOZ}$ and YOZ planes for the variant S5. The inset shows the same in a logarithmic scale.

\section{REFERENCES}

[1] P. Chen, J. Irwin, T.W. Markiewicz, T. Raubenheimer, R. Ruth, P. Wilson, D. Yeremian, in Proc. of the 1996 DPF/DPB Summer Study on New Directions for HighEnergy Physics, Snowmass '96, p. 360.

[2] D. Yu, J. Kim, P. Wilson, in AIP Conf. Proc., Advanced Accelerator Concepts, AIP 279 (1992)p.85.

[3] D. Yu, P. Wilson, in Proc. of the 1993 Particle Accelerator Conf., Washington D.C., May 1993, p. 2681.

[4] D. Yu, S. Ben-Menahem, P. Wilson, R. Miller, R. Ruth, A. Nassiri, in AIP Conf. Proc., Advanced Accelerator Concepts, AIP 335 (1994), p. 800.

[5] D. U. L. Yu and A. V. Smirnov, in AIP Conf. Proc., Advanced Accelerator Concepts AIP 569 (2000)

[6] W. Bruns, in Proc. of Particle Accelerator Conf. (PAC'97), Vancouver, B.C., Canada 12-16 May 1997, p. 2651. 